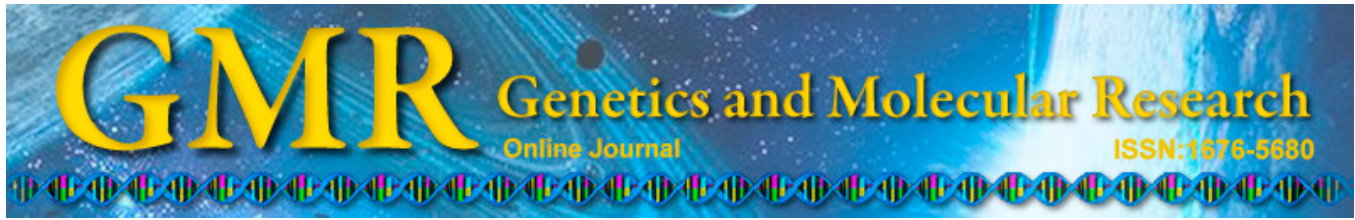

\title{
Expression profiles of retinoic acid synthetases ALDH1As and metabolic enzymes CYP26s in adult and embryonic zebrafish (Danio rerio)
}

\author{
J. Xi' ${ }^{2}$ J. Yue ${ }^{1}$ and Z. Yang ${ }^{1}$ \\ ${ }^{1}$ Department of Pharmacology, \\ Wuhan University School of Basic Medical Science, Hubei, China \\ ${ }^{2}$ Department of Pharmacy, \\ Wuhan University of Science and Technology School of Medicine, Hubei, China \\ Corresponding author: Z. Yang \\ E-mail: zheqiong_yang@whu.edu.cn \\ Genet. Mol. Res. 14 (2): 3948-3956 (2015) \\ Received May 23, 2014 \\ Accepted October 29, 2014 \\ Published April 27, 2015 \\ DOI http://dx.doi.org/10.4238/2015.April.27.9
}

\begin{abstract}
Retinoic acid (RA) plays a crucial role in cellular proliferation, differentiation, and apoptosis. The physiological activity of RA begins early in development and continues throughout an organism's life. RA distribution is tightly controlled by the RA synthetases ALDH1As and the metabolic enzymes CYP26s. We analyzed the expressions of ALDH1As and CYP26s in whole embryos during zebrafish (Danio rerio) development and in adult zebrafish organs, by quantitative reverse transcriptase polymerase chain reaction analysis. All the ALDH1A and CYP26 genes exhibited similar pulse expression patterns, with peak expressions at different developmental stages. ALDH1A2 exhibited an earlier and sharper expression peak [12 hours post-fertilization (hpf)] than ALDH1A3 (24 hpf). CYP26A1 transcription peaked earlier (8 hpf) than CYP26B1 and CYP26Cl (12 hpf), while $C Y P 26 C l$ expression dropped to basal levels later (48 hpf) than that of CYP26A1 and CYP26B1 (18 hpf). ALDH1A2 and CYP26A1 exhibited the highest mRNA peak level and seem to be the dominant
\end{abstract}


isoenzymes in their families during zebrafish development. Expression patterns of ALDH1As and CYP26s in most adult zebrafish tissues were similar to those in humans. Nevertheless, three CYP26s were more vigorously expressed in the zebrafish brain than in human organs, whereas much weaker ALDH1A and CYP26 transcription was found in the zebrafish liver and intestine. This suggests that RA metabolic rates differ between zebrafish and humans or that other enzymes are responsible for RA homeostasis in the zebrafish liver and intestine. All the ALDH1A and CYP26 genes exhibited distinct expression patterns during zebrafish development and in adult zebrafish tissues.

Key words: ALDH1A; CYP26; Retinoic acid; Zebrafish

\section{INTRODUCTION}

Retinoic acid (RA), the primary metabolic derivative of vitamin A (retinol), is one of the candidate molecules hailed as a "morphogen" (Lewandoski and Mackem, 2009). Numerous studies have revealed that a broad range of physiological processes, from embryonic development to adult homeostasis, is regulated by RA. During embryonic development, RA regulates axial and regional patterning, organogenesis, limb formation, and neurogenesis (Campo-Paysaa et al., 2008; Rhinn and Dolle, 2012). During adulthood, RA is involved in organ homeostasis, tissue and organ regeneration, and the prevention of neoplastic growth and neurodegenerative diseases (Maden, 2007; Alvarez et al., 2011). RA is also currently used in the treatment of some cancers (Tang and Gudas, 2011). At the molecular level, RA carries out its diverse biological functions by regulating gene expression, either directly or through indirect cascades. RA controls the expression of hundreds of genes, including transcription factors, enzymes, structural proteins, cell-surface receptors, neurotransmitters, neuropeptide hormones, and growth factors (Balmer and Blomhoff, 2002). In responding cells, RA serves as a ligand for two families of nuclear receptors: RA receptors (RARs) and retinoid X receptors (RXRs) (Mark et al., 2006). The RA:RAR/RXR complex binds to RA response elements within the promoters of target genes and recruits coactivators, thereby bringing about transcriptional changes, and regulates cell proliferation, differentiation, and apoptosis (Kiss et al., 2013).

The level of RA present in a given tissue is tightly controlled by the metabolic machinery of synthesis and degradation, setting up a pre-receptor system that directs the timing and tissue specificity of RA signaling (Kam et al., 2012). RA is synthesized by the sequential oxidation of retinol. The first step in the synthesis of RA is the reversible conversion of retinol to retinal, which is catalyzed by retinol or alcohol dehydrogenases. The second step is the irreversible and rate-limiting conversion of retinal to RA, where the oxidation is mediated by aldehyde dehydrogenases (ALDH1As), also known as retinaldehyde dehydrogenases. There are three ALDH1 As (1, 2, and 3) in mammals (Duester, 2008), and ALDH1A2 and ALDH1A3 are expressed in zebrafish (Danio rerio). RA degradation is controlled by the cytochrome P450 family, chiefly the CYP26 subfamily (CYP26A1, CYP26B1, and CYP26C1) (Ross and Zolfaghari, 2011). All of the members of the CYP26 family are expressed in humans, rodents, and zebrafish. These enzymes catalyze the oxidation of RA into a wide variety of more polar metabolites for excretion. During embryonic development, either an excess or paucity of RA can result in defects and malformations in the craniofacial, central nervous, and cardiovascular 
systems of the fetus (McCaffery et al., 2003; Keyte and Hutson, 2012). In adults, an abnormal level of RA can cause impaired vision, skin diseases, and dysfunction of the nervous system (Sieving et al., 2001; Cheepala et al., 2007; Maden, 2007). Therefore, the precise spatio-temporal control of ALDH1A and CYP26 expression during embryogenesis and in adult tissues is critical to define the proper tissue exposure to RA, and is an essential aspect of normal morphogenesis and function maintenance in adult organs.

The zebrafish is an important model organism that is used in molecular genetics and developmental biology. It serves as a model for understanding normal vertebrate development, as well as for investigating the mechanisms underlying human diseases. In this study, we analyzed the expression patterns of two enzyme families, the RA synthetases ALDH1As and the metabolic enzymes CYP26s, in the whole embryo and the adult tissues of zebrafish using quantitative reverse transcriptase polymerase chain reaction (qRT-PCR) analysis. This is the first quantitative characterization of ALDH1 A and CYP26 expression in zebrafish whole embryos at various developmental stages, and in a complete set of adult zebrafish tissues.

\section{MATERIAL AND METHODS}

\section{Fish maintenance}

Adult and embryonic zebrafish were handled in compliance with local animal care regulations. A wild-type $(\mathrm{AB})$ strain was maintained at $28^{\circ} \mathrm{C}$ under a $14 \mathrm{~h} \mathrm{light} / 10 \mathrm{~h}$ dark cycle. Embryos were collected by natural spawning, raised in Petri dishes, and staged according to Kimmel et al. (1995). Embryonic age was expressed as the number of hours postfertilization (hpf).

\section{Total RNA isolation and qRT-PCR}

We prepared three total RNA samples for each developmental stage of the zebrafish embryo $(0,2,4,8,12,18,24,48$, and $72 \mathrm{hpf})$, and each tissue of the adult zebrafish (brain, heart, liver, kidney, spleen, eye, intestine, muscle, swim bladder, and gills). For each sample, total RNA was isolated from 100 whole embryos and each adult tissue from three males and three females that were 8 months old, and pooled into one sample for RNA isolation. The testes of six males or the ovaries of six females were pooled for one sample. An RNAiso Plus kit (Takara, Dalian, China) was used, according to manufacturer instructions. First-strand cDNA was synthesized using a RevertAid First-Strand cDNA Synthesis kit (Thermo Scientific, Beijing, China) and random primers (Promega, Beijing, China) under the following conditions: $65^{\circ} \mathrm{C}$ for $5 \mathrm{~min}, 42^{\circ} \mathrm{C}$ for $60 \mathrm{~min}$, and $70^{\circ} \mathrm{C}$ for $5 \mathrm{~min}$. The qPCR analysis was conducted using iTaq $^{\text {TM }}$ Universal SYBR ${ }^{\circledR}$ Green Supermix (Bio-Rad, Shanghai, China). The qPCR thermal cycler conditions were as follows: initial denaturation at $95^{\circ} \mathrm{C}$ for $3 \mathrm{~min}$, followed by 40 cycles of $30 \mathrm{~s}$ at $95^{\circ} \mathrm{C}, 30 \mathrm{~s}$ at $60^{\circ} \mathrm{C}$, and $20 \mathrm{~s}$ at $72^{\circ} \mathrm{C}$. Each experiment was performed three times, and each PCR amplification was performed in triplicate. The relative abundance of the target cDNA was calculated from a serially diluted cDNA pool (standard curve), with Bio-Rad CFX Manager V1.6 software, using the $2^{-\Delta \Delta C t}$ method. The data were normalized against GAPDH expression. Genes were considered significantly expressed when their relative expression value compared to GAPDH was higher than $0.5(\mathrm{P}<0.05)$. The gene-specific sense and antisense primers are shown in Table 1. 


\section{RESULTS}

\section{Multiple protein sequence alignments of CYP26s and ALDH1As in zebrafish, mouse, and human}

A detailed comparison of the zebrafish, mouse, and human ALDH1A and CYP26 protein sequences was performed, according to the sequences available from the NCBI GenBank database. As shown in Figure 1, zebrafish ALDH1A2 and ALDH1A3 proteins exhibited a high sequence homology with mice (78 and $71 \%$, respectively) and humans (80 and $71 \%$, respectively).

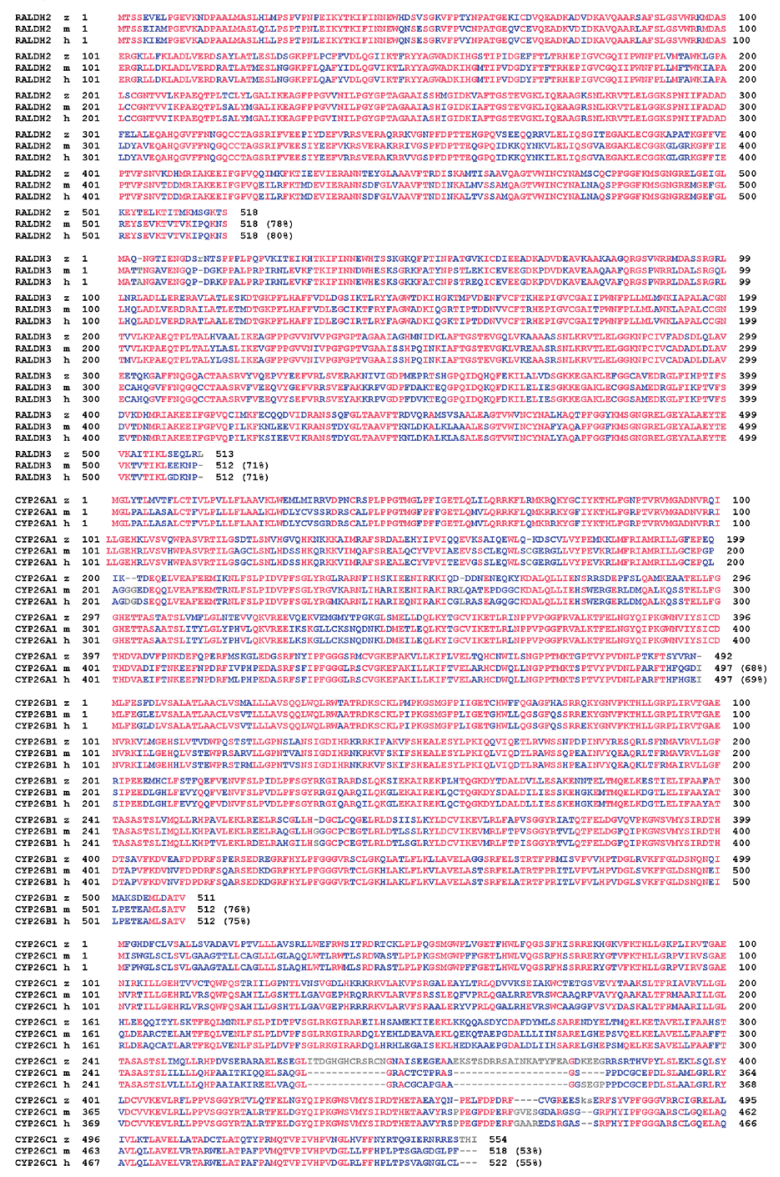

Figure 1. Multiple protein sequence alignments of zebrafish CYP26s and ALDH1As with mouse and human. Multiple sequence alignment columns are colored in blue or red. The red color indicates identical residues, and blue indicates non-conservative ones. Numbers in parentheses at the end of mouse and human sequences show the percentage of identity of mouse and human sequence to zebrafish sequence, respectively. GenBank accession numbers of these protein sequences are as follows: zebrafish CYP26A1, NP 571221.2; mouse CYP26A1, NP_031837.2; human CYP26A1, NP_000774.2; zebrafish CYP26B1, NP 997831.1; mouse CYP26B1, NP 001171184.1; human CYP26B1, NP 063938.1; zebrafish CYP26C1, NP 001025122.2; mouse CYP26C1, NP ${ }^{-}$001098671.1; human CYP26C1, NP 899230.2; zebrafish ALDH1A2, NP 571925.1; mouse ALDH1A2, NP_033048.2; human ALDH1A2, NP_003879.2; zebrafish ALDH1A3, NP_001038210.1; mouse ALDH1A3, NP_444310.3; human ALDH1A3, NP_000684.2. 
Zebrafish CYP26A1, CYP26B1, and CYP26C1 proteins were also highly homologous with mouse $(68,76$, and $53 \%$, respectively) and human $(69,75$, and $55 \%$, respectively) amino acid identity. Therefore, zebrafish ALDH1A and CYP26 proteins, and their mouse and human orthologs, were highly conserved during evolution, which indicates their essential function in these animals. Moreover, within individual enzyme families, the ALDH1A2 protein is $71 \%$ homologous with the ALDH1A3 protein, and the proteins encoded by three zebrafish CYP26 genes also exhibit $41 \%$ sequence homology. Despite a high degree of sequence similarity, each gene in the CYP26 and ALDH1A families was expressed in a clearly distinguishable pattern, as described below.

\section{Developmental expression patterns of ALDH1As and CYP26s in zebrafish whole embryos}

To study the expression patterns of zebrafish ALDH1As and CYP26s during embryonic development, we performed qRT-PCR analysis of the total embryonic RNA from the zygote to $72 \mathrm{hpf}$. The primer sequences are listed in Table 1. Interestingly, we found that all of the genes in the ALDH1A and CYP26 families exhibited similar pulse expression patterns, with peak expressions at different developmental stages (Figure 2). In the ALDH1A family, ALDH1A2 transcription was not detectable, or was at a very low level, until the $75 \%$-epiboly stage $(8 \mathrm{hpf})$. It then started to increase, and quickly reached a peak at the 9 -somite stage (12 hpf). Before its expression dropped to basal levels at the long-pec stage (48 hpf), there was a small increase at the prim-5 stage ( $24 \mathrm{hpf}$ ), but to a lesser extent than in CYP26B1. The ALDH1A3 mRNA level started to increase at the same developmental stage ( $8 \mathrm{hpf}$ ) as ALDH1A2, but more slowly. After the peak was reached at the prim-5 stage ( $24 \mathrm{hpf})$, ALDH1A3 transcription immediately decreased, and fell to baseline levels at the long-pec stage (48 hpf). It seems that ALDH1A2 is the dominant form in the ALDH1A family during zebrafish development, since the relative amount of ALDH1A2 transcripts at peak level (12 hpf) was more than 8-fold higher than that of ALDH1A3 transcripts ( $24 \mathrm{hpf}$ ). Moreover, the mRNA copy number of ALDH1A3 at peak level (24 hpf) was in the same order of magnitude as ALDH1A2 transcripts at the second small peak (24 hpf).

In the CYP26 family, the qRT-PCR results revealed the presence of all of the CYP26 gene transcripts at all the developmental stages we analyzed, including the zygote, which indicates that they are also maternally expressed. CYP26A1 was expressed at a low level until the sphere stage (4 hpf), then quickly increased and reached a peak at the $75 \%$-epiboly stage ( $8 \mathrm{hpf})$, which was maintained until the 9 -somite stage (12 hpf). Its expression decreased at the 19-20-somite stage (18 hpf), and dropped to basal levels at the long-pec stage (48 hpf). CYP26B1 and CYP26Cl transcripts were also detectable from the 1-cell (0 hpf) to the $75 \%$-epiboly stages ( $8 \mathrm{hpf}$ ) at a lower level than $C Y P 26 \mathrm{Al}$, and then increased and reached a peak later than CYP26A1, at the 9-somite stage (12 hpf). A rapid decrease in CYP26B1 expression started at the 9 -somite stage $(12 \mathrm{hpf})$, which was followed by a small increase at the prim-5 stage ( $24 \mathrm{hpf})$. It decreased to basal levels at the long-pec stage (48 hpf), which was similar to that observed in $A L D H 1 A 2$. In contrast, $C Y P 26 C 1$ transcription gradually decreased after the 9-somite stage (12 hpf), before reaching basal levels at the long-pec stage (48 hpf). Of these three CYP26 isoenzymes, CYP26A1 seems to be the dominant isoform during zebrafish development, since its expression stayed at a high level for a longer period than did the other two CYP26 genes, and the relative copy number of CYP26A1 transcripts at the peak level (8 
hpf) was approximately 3.5 -fold higher than that of CYP26B1 (12 hpf), and 1.8-fold higher than that of CYP26C1 (12 hpf). In addition, the CYP26A1 mRNA copy number at basal levels before the peak was more than 10-fold higher than that of CYP26B1 or CYP26C1.

\begin{tabular}{|c|c|c|}
\hline Gene & Direction & Sequences \\
\hline \multirow{2}{*}{ ALDH1A2 } & Forward & 5'-CAGGAGAGGTGAAGAACGACC-3' \\
\hline & Reverse & 5'-AACCCAGAGAGAAGGCCGAA-3' \\
\hline \multirow{2}{*}{$A L D H 1 A 3$} & Forward & 5'-GCTATGGCACAGAACGGGAC-3' \\
\hline & Reverse & 5'-TTAGCAGCTTTCACCGCCTC-3' \\
\hline \multirow{2}{*}{ CYP26A1 } & Forward & 5'-CAGAGAGGAGGTTCAGGAGA-3' \\
\hline & Reverse & 5'-GCGTGTCACAGATGCTGTAAAT-3' \\
\hline \multirow[t]{2}{*}{ CYP26BI } & Forward & 5'-ATGGCACTTCTTCTGGCCGT-3' \\
\hline & Reverse & 5'-CTTACGCACATTCTCTGCCCC-3' \\
\hline \multirow{2}{*}{ CYP26Cl } & Forward & 5'-GTCTTACCGACAGTGCTGCT-3' \\
\hline & Reverse & 5'-TGTTTTCTGCACCGGTCACT-3' \\
\hline \multirow{2}{*}{$G A P D H$} & Forward & 5'-TCACATTAAGGGTGGTGCAA-3' \\
\hline & Reverse & 5'-GTGATGGCATGAACAGTGCT-3' \\
\hline
\end{tabular}
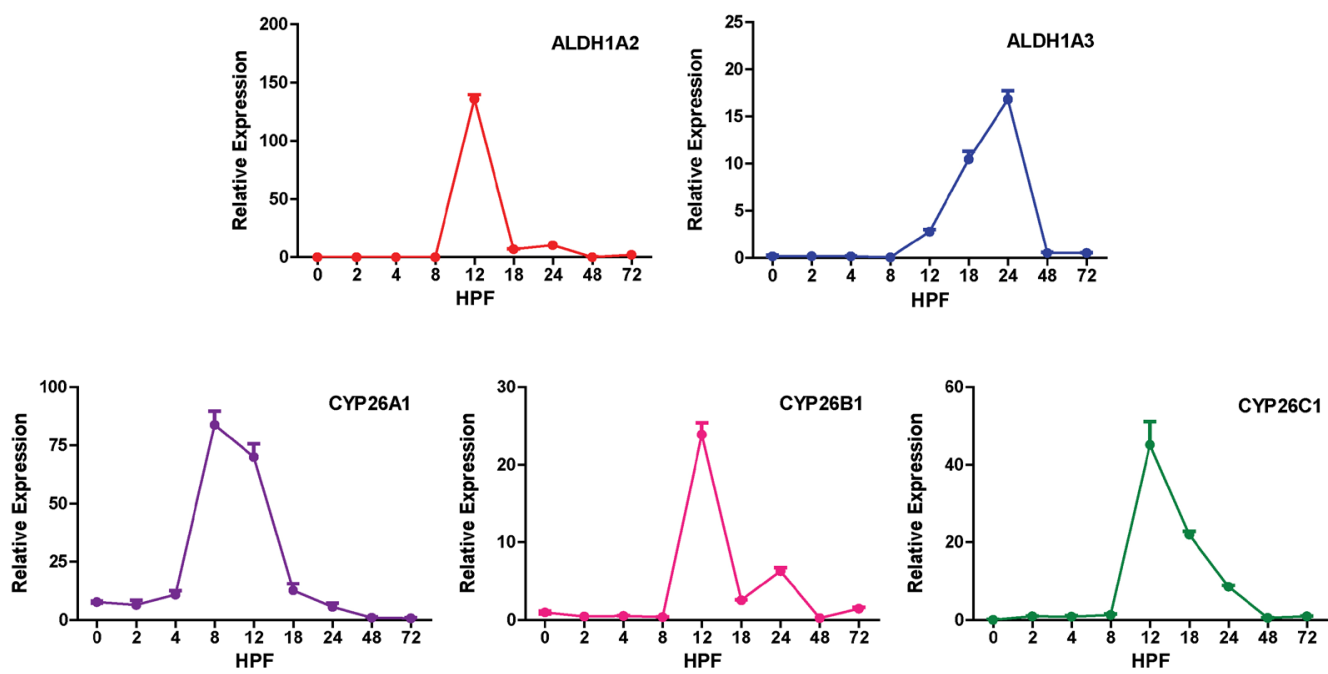

Figure 2. Developmental expression patterns of ALDH1As and CYP26s in zebrafish whole embryos. ALDH1As and CYP26s expression during zebrafish development was measured by qRT-PCR performed on RNA extracted from whole embryos from 1-cell to $72 \mathrm{hpf}$ stages, and shown as relative expression compared to GAPDH levels.

\section{Expression profiles of ALDH1As and CYP26s in adult zebrafish tissues}

A detailed characterization of ALDH1A and CYP26 expression in adult zebrafish organs was performed using qRT-PCR analysis. As shown in Figure 3, ALDH1A2 was vigorously expressed in the spleen, and was moderately expressed in the brain and testes. Its expression was absent in the heart, liver, kidneys, and ovaries, and a very low level of $A L D H 1 A 2$ mRNA was detected in the rest of the tissues examined. Compared to ALDH1A2, ALDH1A3 expression was broader, but the level was relatively lower. A strong expression of $A L D H 1 A 3$ was found in the brain, eyes, and gills, while a relatively low level of mRNA was detected in 
the spleen and muscle. $A L D H 1 A 3$ mRNA was not detectable in the heart, liver, intestine, or ovaries, and the rest of the tissues exhibited very low expression levels.

Similar to the ALDH1A family, the expression patterns of CYP26A1, CYP26B1, and $C Y P 26 C 1$ differed from each other. CYP26A1 was highly expressed in the brain and eye, moderately expressed in the spleen, testes, and gills, and not detectable in the heart or liver, while the rest of the tissues exhibited very low levels of expression. CYP26B1 was also strongly expressed in the brain; it was the most abundantly expressed CYP26 in this tissue. CYP26B1 mRNA was less abundant in the spleen, eyes, testes, muscle, and gills, while its expression levels in the kidney and swim bladder were low, and no significant transcription was detected in the heart, liver, intestine, or ovaries. CYP26Cl expression seemed to be the broadest of all three zebrafish CYP26s; high levels of transcription were detected in the brain, spleen, and muscle, while moderate transcription levels were found in the eyes, testes, swim bladder, and gills. The kidneys and ovaries exhibited a very low level of CYP26Cl expression, and no significant expression was detected in the heart, liver, or intestine.
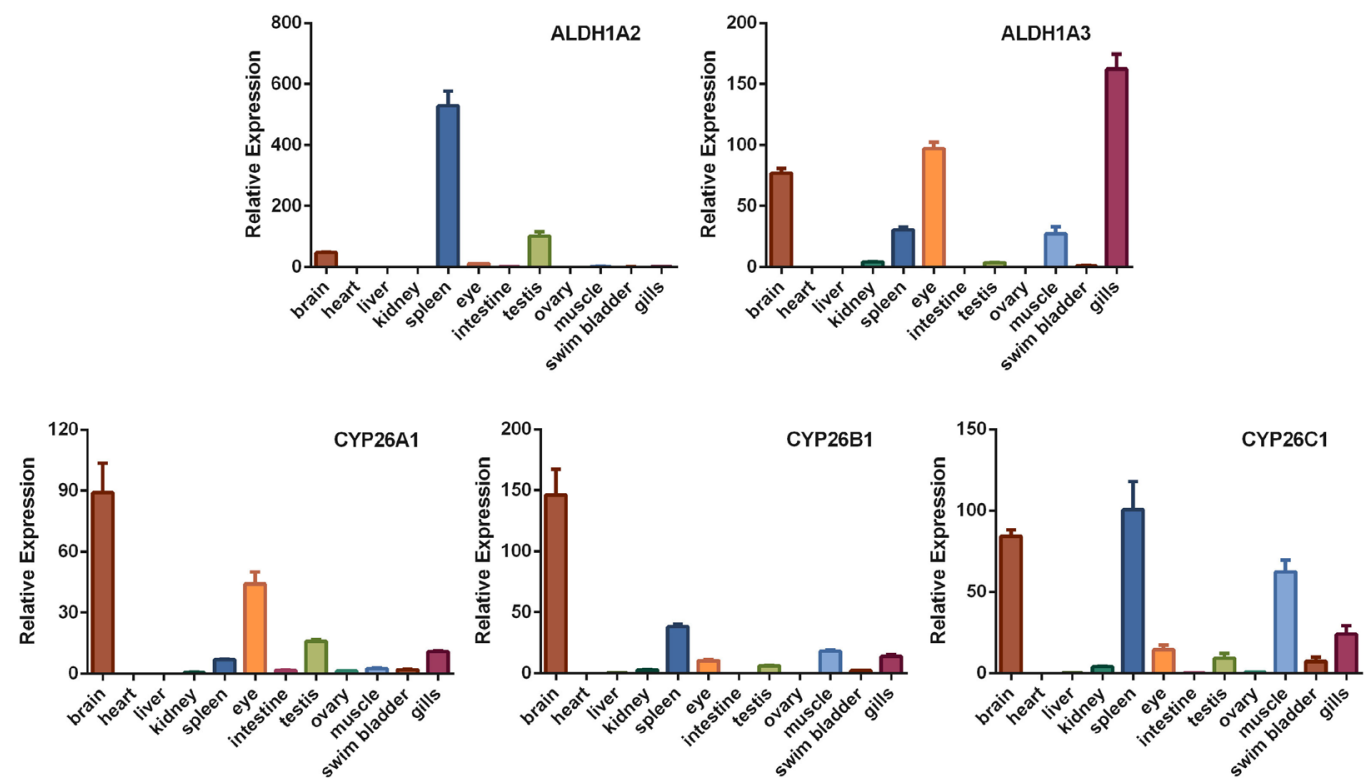

Figure 3. Expression profiles of ALDH1As and CYP26As in zebrafish adult tissues. CYP26A1, CYP26B1, CYP26C1, ALDH1A2 and ALDH1A3 transcripts were detected by qRT-PCR by using RNA extracted from zebrafish adult tissues, and shown as relative expression compared to GAPDH levels.

The two ALDH1A genes and the three CYP26 genes shared similarities, but also exhibited significant differences, in the timing as well as in the level of their expression during zebrafish development, and in adult zebrafish tissues.

\section{DISCUSSION}

Our results show that $A L D H 1 A 2$ initiated expression earlier than $A L D H 1 A 3$ during zebrafish development, which is similar to the developmental expression patterns of ALDH1As in the mouse. ALDH1A2 is first expressed at embryonic day 7.5 (E7.5) in the trunk 
presomitic mesoderm in mouse embryos, while $A L D H 1 A 3$ expression is first observed from E8.75 to E9.5 in the optic vesicle and nasal placode (Niederreither et al., 1997; Li et al., 2000). CYP26A1 initiated a high level of expression at an earlier stage of zebrafish embryonic development (4 hpf) than CYP26B1 and CYP26C1 (8 hpf). These results are consistent with CYP26s expression patterns during murine embryonic development. In mouse embryos, CYP26A1 is expressed as early as embryonic day 6.0 (E6.0) in the extraembryonic and embryonic endoderm, just before the gastrulation stage, presumably to protect the embryo from excessive maternal vitamin A or RA, whereas the expression of CYP26B1 is initiated at E8.0 in the hindbrain (Fujii et al., 1997; MacLean et al., 2001).

The expression patterns of ALDH1As and CYP26s in most adult zebrafish tissues, particularly in the heart, kidney, spleen, testes, ovaries, and muscle, are similar to those in humans (Xi and Yang, 2008). Nevertheless, all of the members of ALDH1 A and CYP26 exhibited much lower expression levels in the liver and intestine in the zebrafish, whereas $A L D H 1 A 3$ and CYP26A1 are highly expressed in the human liver, and the expression of ALDH1A3 and CYP26B1 is remarkably strong in the human intestine. This may suggest that the metabolic rate of RA might be lower in the liver and intestine of the zebrafish. Another possible explanation for these differences is that there might be other enzyme families responsible for RA synthesis and metabolism in these organs. For example, CYP1B1 and ALDH8A1 have been reported to be responsible for the ALDH1A-independent generation of RA during vertebrate embryogenesis (Chambers et al., 2007). Some isoenzymes of the CYP3A and CYP2C families have also been reported to exhibit RA-4-hydroxylation activity (Ross and Zolfaghari, 2011). However, the three CYP26 genes seem to be more abundantly expressed in the zebrafish brain than in the human brain, which may suggest a higher RA metabolic rate in the zebrafish brain.

In conclusion, the conserved amino acid sequences of ALDH1As and CYP26s in vertebrates suggests that these two enzyme families are essential for survival, whereas the differences in their spatio-temporal expression patterns suggests a complementary effect of isoenzymes within each family of ALDH1As and CYP26s, and between them.

\section{ACKNOWLEDGMENTS}

Research supported by a Hubei Province Department of Education Outstanding Young Investigator Grant (\#Q20121116) and the National Natural Science Foundation of China (\#31271327). We thank the China Zebrafish Resource Center for technical support.

\section{REFERENCES}

Alvarez S, Bourguet W, Gronemeyer H and de Lera AR (2011). Retinoic acid receptor modulators: a perspective on recent advances and promises. Expert Opin. Ther. Pat. 21: 55-63.

Balmer JE and Blomhoff R (2002). Gene expression regulation by retinoic acid. J. Lipid Res. 43: 1773-1808.

Campo-Paysaa F, Marletaz F, Laudet V and Schubert M (2008). Retinoic acid signaling in development: tissue-specific functions and evolutionary origins. Genesis 46: 640-656.

Chambers D, Wilson L, Maden M and Lumsden A (2007). RALDH-independent generation of retinoic acid during vertebrate embryogenesis by CYP1B1. Development 134: 1369-1383.

Cheepala SB, Syed Z, Trutschl M, Cvek U, et al. (2007). Retinoids and skin: microarrays shed new light on chemopreventive action of all-trans retinoic acid. Mol. Carcinog. 46: 634-639.

Duester G (2008). Retinoic acid synthesis and signaling during early organogenesis. Cell 134: 921-931.

Fujii H, Sato T, Kaneko S, Gotoh O, et al. (1997). Metabolic inactivation of retinoic acid by a novel P450 differentially expressed in developing mouse embryos. EMBO J. 16: 4163-4173. 
Kam RK, Deng Y, Chen Y and Zhao H (2012). Retinoic acid synthesis and functions in early embryonic development. Cell Biosci. 2: 11.

Keyte A and Hutson MR (2012). The neural crest in cardiac congenital anomalies. Differentiation 84: 25-40.

Kimmel CB, Ballard WW, Kimmel SR, Ullmann B, et al. (1995). Stages of embryonic development of the zebrafish. Dev. Dyn. 203: 253-310.

Kiss M, Czimmerer Z and Nagy L (2013). The role of lipid-activated nuclear receptors in shaping macrophage and dendritic cell function: From physiology to pathology. J. Allergy Clin. Immunol. 132: 264-286.

Lewandoski M and Mackem S (2009). Limb development: the rise and fall of retinoic acid. Curr. Biol. 19: R558-561.

Li H, Wagner E, McCaffery P, Smith D, et al. (2000). A retinoic acid synthesizing enzyme in ventral retina and telencephalon of the embryonic mouse. Mech. Dev. 95: 283-289.

MacLean G, Abu-Abed S, Dolle P, Tahayato A, et al. (2001). Cloning of a novel retinoic-acid metabolizing cytochrome P450, Cyp26B1, and comparative expression analysis with Cyp26A1 during early murine development. Mech. Dev. 107: 195-201.

Maden M (2007). Retinoic acid in the development, regeneration and maintenance of the nervous system. Nat. Rev. Neurosci. 8: 755-765.

Mark M, Ghyselinck NB and Chambon P (2006). Function of retinoid nuclear receptors: lessons from genetic and pharmacological dissections of the retinoic acid signaling pathway during mouse embryogenesis. Annu. Rev. Pharmacol. Toxicol. 46: 451-480.

McCaffery PJ, Adams J, Maden M and Rosa-Molinar E (2003). Too much of a good thing: retinoic acid as an endogenous regulator of neural differentiation and exogenous teratogen. Eur. J. Neurosci. 18: 457-472.

Niederreither K, McCaffery P, Drager UC, Chambon P, et al. (1997). Restricted expression and retinoic acid-induced downregulation of the retinaldehyde dehydrogenase type 2 (RALDH-2) gene during mouse development. Mech. Dev. 62: 67-78.

Rhinn M and Dolle P (2012). Retinoic acid signalling during development. Development 139: 843-858.

Ross AC and Zolfaghari R (2011). Cytochrome P450s in the regulation of cellular retinoic acid metabolism. Annu. Rev. Nutr. 31: 65-87.

Sieving PA, Chaudhry P, Kondo M, Provenzano M, et al. (2001). Inhibition of the visual cycle in vivo by 13-cis retinoic acid protects from light damage and provides a mechanism for night blindness in isotretinoin therapy. Proc. Natl. Acad. Sci. U S A 98: 1835-1840.

Tang XH and Gudas LJ (2011). Retinoids, retinoic acid receptors, and cancer. Annu. Rev. Pathol. 6: 345-364.

Xi J and Yang Z (2008). Expression of RALDHs (ALDH1As) and CYP26s in human tissues and during the neural differentiation of P19 embryonal carcinoma stem cell. Gene. Expr. Patterns 8: 438-442. 